\title{
COVID-19 in Liver Transplant Recipients - A Series with Successful Recovery
}

\author{
Ashok Choudhury ${ }^{1}$, Golamari Srinivasa Reddy ${ }^{1}$, Shantan Venishetty ${ }^{1}$, Viniyendra Pamecha ${ }^{2}$, \\ Saggere Muralikrishna Shasthry ${ }^{1}$, Arvind Tomar $^{3}$, Lalita Gauri Mitra ${ }^{4}$, \\ Venkata Siva Tez Prasad ${ }^{5}$, Rajendra Prasad Mathur ${ }^{5}$, Debajyoti Bhattacharya ${ }^{3}$ \\ and Shiv Kumar Sarin*1
}

${ }^{1}$ Department of Hepatology and Liver Transplant, Institute of Liver and Biliary Sciences, New Delhi, India; ${ }^{2}$ Department of HPB Surgery and Liver Transplant, Institute of Liver and Biliary Sciences, New Delhi, India; ${ }^{3}$ Department of Pulmonary Medicine, Institute of Liver and Biliary Sciences, New Delhi, India; ${ }^{4}$ Department of Critical Care Medicine, Institute of Liver and Biliary Sciences, New Delhi, India; ${ }^{5}$ Department of Nephrology, Institute of Liver and Biliary Sciences, New Delhi, India

\begin{abstract}
The severe acute respiratory syndrome corona virus-2 (referred to as SARS-CoV-2) pandemic had a great impact on public life in general as well as on populations with preexisting disease and co-morbidities. Liver transplant and immunosuppressant medication predisposes to more severe disease and is often associated with poor outcome. The clinical features, disease course, treatment and process of modulating the immunosuppression is challenging. Here, we describe the clinical presentation, treatment and outcomes in six liver transplant recipients. Out of those six patients, three had mild, one had moderate and one had severe COVID-19, and one was asymptomatic. The immunosuppression minimization or withdrawal was done based upon the clinical severity. Consideration of tocilizumab and/or convalescent plasma as well as antivirals i.e. remdesvir done in severe cases. The routine practice of prophylactic anticoagulation, consideration of repurposed drugs (i.e. teicoplanin and doxycycline), and watchful monitoring of asymptomatic recipients helped to achieve an uneventful recovery.

Citation of this article: Choudhury A, Reddy GS, Venishetty S, Pamecha V, Shasthry SM, Tomar A, et al. COVID19 in liver transplant recipients - A series with successful recovery. J Clin Transl Hepatol 2020;8(4):467-473. doi: 10.14218/JCTH.2020.00061.
\end{abstract}

\section{Introduction}

A cumulative total of nearly 25 million cases and 800000 deaths have been reported since the start of the outbreak of severe acute respiratory syndrome corona virus-2

Keywords: Liver transplant; COVID-19; SARS-CoV-2; Liver injury; Remdesivir; Convalescent plasma.

Abbreviations: COVID-19, corona virus disease 2019; LDLT, live donor liver transplant; LT, liver transplant; SARS-CoV-2, severe acute respiratory syndrome corona virus- 2 .

Received: 7 July 2020; Revised: 28 August 2020; Accepted: 29 August 2020

*Correspondence to: Shiv Kumar Sarin, Department of Hepatology and Liver Transplant, Institute of Liver and Biliary Sciences, New Delhi 110070, India. E-mail: shivsarin@gmail.com
(SARS-CoV-2). ${ }^{1}$ The majority of cases often present with mild symptoms, like fever, cough and shortness of breath; however, the severity of symptoms increases with presence of co-morbidities and pre-existing diseases, such as the presence of chronic liver disease. ${ }^{2}$ The data on immunosuppression therapy, post-transplant status and impact of SARS-CoV-2 infection on a liver graft as well as the overall survival in liver graft recipients is largely inadequate. A similar lack of information is present regarding the treatment, drug interaction and overall outcome with solid organ transplant and corona virus disease 2019 (COVID-19).

Recently published data from the European Liver Transplant Registry (ELTR) of 103 cases showed high mortality (i.e. $16 \%)$, with a much higher rate among those on ventilator support (44\%), above 60 years of age, and transplanted at 2 years or more before the COVID- 19 disease. ${ }^{3}$ To publication, more than 540,000 confirmed cases of COVID-19, with 16,475 deaths, have been reported from India. ${ }^{1}$ Every year, on average more than 1500 liver transplants (LTs) are carried out in India, but the effects of infection with SARS-CoV-2 and the implications of such are not yet reported. ${ }^{4}$ One study was conducted on patients admitted to Institute of Liver and Biliary Sciences (ILBS) New Delhi, India, which is a highvolume transplant center ( $>100$ cases per year), working with live donors mainly. Today, there is a pandemic situation, with nearly 150,000 active cases and a seroprevalence of COVID-19 at $23 \%$ in the population. ${ }^{5}$

This present series of six LT recipients, all within the spectrum of presentation (i.e. asymptomatic, mild, moderate and severe cases), were managed well and recovered successfully.

\section{Case series}

The present series of six cases include one severe, one moderate and three mild COVID-19 diseases, and one asymptomatic SARS-CoV-2 infection. The clinical characteristics (Table 1) and laboratory data (Table 2), along with the timeline for maintenance immunosuppression and COVID19-specific treatments and outcome are shown. Diagnosis was performed by nasopharyngeal swab test with RT-PCR. Chest radiography and high-resolution computed tomography were performed selectively, upon diagnosis of moderate 
and severe COVID-19 disease as per World Health Organization definition. All the patients were managed in a dedicated COVID-19 ward with intensive care unit facility at Institute of Liver and Biliary Sciences.

\section{Severe COVID-19 in a post-LT recipient}

A 52 year-old gentleman, post-live donor (LD)LT for decompensated nonalcoholic steatohepatitis cirrhosis ( 8 months back) presented with high-grade fever, cough (for 4 days), and increasing shortness of breath (for 1 day). At admission, he was febrile with temperature of $99^{\circ} \mathrm{F}$, oxygen saturation of $92 \%$ at room air, respiratory rate of 22 breaths $/ \mathrm{m}$, blood pressure of $120 / 80 \mathrm{mmHg}$, and pulse rate of $112 / \mathrm{min}$. Chest $\mathrm{X}$-ray showed bilateral lower lobe infiltrations. Chest computed tomography revealed mixed diffuse ground-glass opacities with multifocal patchy consolidations involving both lungs, consistent with atypical infection likely due to SARSCoV-2 (Fig. 1). The laboratory results (Table 2 ) reflected total normal leukocyte count with lymphopenia ( $8 \%)$, high neutrophil-to-lymphocyte ratio 10 and thrombocytopenia $\left(82 \times 10^{3} /\right.$ cc) with nearly normal bilirubin, aspartate transaminase, Alanine transaminase, serum alkaline phosphatase and gamma-glutamyl transpeptidase. After admission to the intensive care unit, his hypoxia required support by noninvasive ventilation and he was started on dexamethasone $(6 \mathrm{mg}$ once daily), loading dosage of tocilizumab $(8 \mathrm{mg} / \mathrm{kg}, 400$ $\mathrm{mg}$ ), hydroxychloroquine (400 mg twice daily), antibiotics (piperacillin-tazobactam and teicoplanin), and prophylactic enoxaparin. Tacrolimus as well as mycophenolate was kept on-hold, temporarily. The dexamethasone at low dose helped in low-dose maintenance for the LT status, in addition to its role in COVID-19. In view of persistent hypoxia (oxygen saturation of $<94 \%$ ), tachypnoea and fever, remdesivir was started ( $200 \mathrm{mg}$ bolus, followed by $100 \mathrm{mg}$ once daily) on the third day with a second dose of tocilizumab, and hydroxychloroquine was stopped. However, on day 7 , he again experienced respiratory distress (oxygen saturation of $90 \%$ ), requiring $10 \mathrm{~L} / \mathrm{m}$ of oxygen on high-flow nasal cannula. The convalescent plasma was planned at admission but deferred due to resource constraint; however, it was able to be transfused on day 8 (delayed, but considered as rescue therapy), along with remdesivir being continued for 10 days. The patient improved over time, his oxygen requirement was maintained with $2 \mathrm{~L}$ of oxygen. X-ray showed improvement, and the fever, neutrophil-to-lymphocyte ratio, and thrombocytopenia improved by the $10^{\text {th }}$ day (Fig. 2). As of day 14 , he was maintaining oxygen saturation at room air, and the tacrolimus with prednisolone treatment was reintroduced without mycophenolate.

\section{Moderate COVID-19 in post-LT recipient}

A 39 year-old gentleman, 6 years from LDLT for decompensated ethanol-related cirrhosis with diabetes, hypertension, obesity and biliary anastomotic stricture (stent-free for 2 years), presented with 3-day history of fever and dry cough. At presentation, he was febrile with temperature of $100^{\circ} \mathrm{F}$, oxygen saturation of $96 \%$ under ambient air, respiratory rate of 22 breaths $/ \mathrm{m}$, blood pressure of $136 / 84 \mathrm{mmHg}$, and pulse rate of $102 / \mathrm{m}$. Chest X-ray showed bilateral lower lobe infiltrations. Chest computed tomography revealed features atypical of pneumonia or viral pneumonia (Fig. 3). The previous immunosuppression regimen (i.e. tacrolimus and mycophenolate) was stopped and the patient was maintained on prednisolone ( $20 \mathrm{mg}$ once daily) and $2 \mathrm{~L} / \mathrm{m}$ oxygen by nasal canula. He received hydroxychloroquine (400 mg twice daily) and prophylactic anticoagulation. However on day 8 of the hospital stay, he had a spiking fever [up to $102^{\circ} \mathrm{F}$, worsening leukopenia (of $2.4 \times 10^{3} / \mathrm{cc}$ ), increasing neutrophil-to-lymphocyte ratio (to 4.8 ), and respiratory distress with oxygen saturation of $90 \%$, requiring $8 \mathrm{~L} / \mathrm{m}$ of oxygen by high-flow nasal cannula]. Therapy with remdesivir, tocilizumab or convalescent plasma was unavailable, so he was started on teicoplanin and doxycycline (100 mg twice daily), with antibiotics upgraded upon suspicion of nosocomial pneumonia, despite culture and procalcitonin showing non-contributory roles. The patient became afebrile with improvement in clinical symptoms in the next $72 \mathrm{~h}$. He was discharged with low-dose tacrolimus ( $0.5 \mathrm{mg}$ twice daily) and prednisolone ( $5 \mathrm{mg}$, once daily starting on the $15^{\text {th }}$ day), and with a plan for resuming previous immunosuppression on follow-up.

\section{Mild COVID-19 in post-LT recipients}

We successfully managed three mild COVID-19 patients with mild disease, and all had a separate background of disease in addition to their post-transplant status. The first one was a 48 year-old male with pre-transplant hypertension, hypothyroidism and receipt of a deceased donor graft 6 years back. He presented with a 2-day history of fever and cough. At admission, he was febrile, oxygen saturation of $98 \%$ under ambient air, respiratory rate of $18 / \mathrm{m}$, with normal chest X-ray findings. The second was a 50 year-old gentleman, who was 7 months post-LDLT with biliary anastomotic stricture and a Percutaneous Transhepatic Billiary Drainage (PTBD) catheter in situ. He presented with a 2-day history of fever and a sore throat. At presentation, his temperature was $99.6^{\circ} \mathrm{F}$, oxygen saturation was $95 \%$ under ambient air, and respiratory rate was 22 breaths/m. Initial suspicion of cholangitis was kept; however, in view of the current pandemic as well his sore throat, we tested for SARS-CoV-2 and obtained a positive result. Antibiotic coverage with meropenem and fluconazole continued, due to deranged Liver function parameters (cholestatic pattern, prolonged PTBD, but a negative bile culture). The tacrolimus was stopped, and the patient was maintained on prednisolone ( $20 \mathrm{mg}$ once daily). The third patient was a 38 year-old gentleman, being 5 years' post-LDLT, who presented with a 2-day history fever and dry cough and a 1day history of shortness of breath. He had a bad post-transplant course with recidivism, graft failure, development of cirrhosis after transplant and chronic kidney disease due to immunoglobulin A nephropathy and maintenance dialysis. At presentation, he was febrile $\left(99.4^{\circ} \mathrm{F}\right)$, oxygen saturation of $92 \%$ under ambient air, respiratory rate of $22 / \mathrm{m}$, and blood pressure of $170 / 90 \mathrm{mmHg}$. Chest X-ray was suggestive of central prominence suggestive of fluid overload, and testing for SARS-CoV-2 gave positive result. Urgent hemodialysis was performed to address metabolic acidosis, high creatinine, anuria and respiratory distress.

All these patients, managed in the COVID-19 ward, received hydroxychloroquine and prophylactic enoxaparin, stoppage of immunosuppression, and were maintained on low-dose prednisolone (10-20 mg per day). The previous immunosuppression was started on day 14 and all had a negative throat swab result for SARS-CoV-2 at discharge. 
Choudhury A. et al: COVID-19 and liver transplant

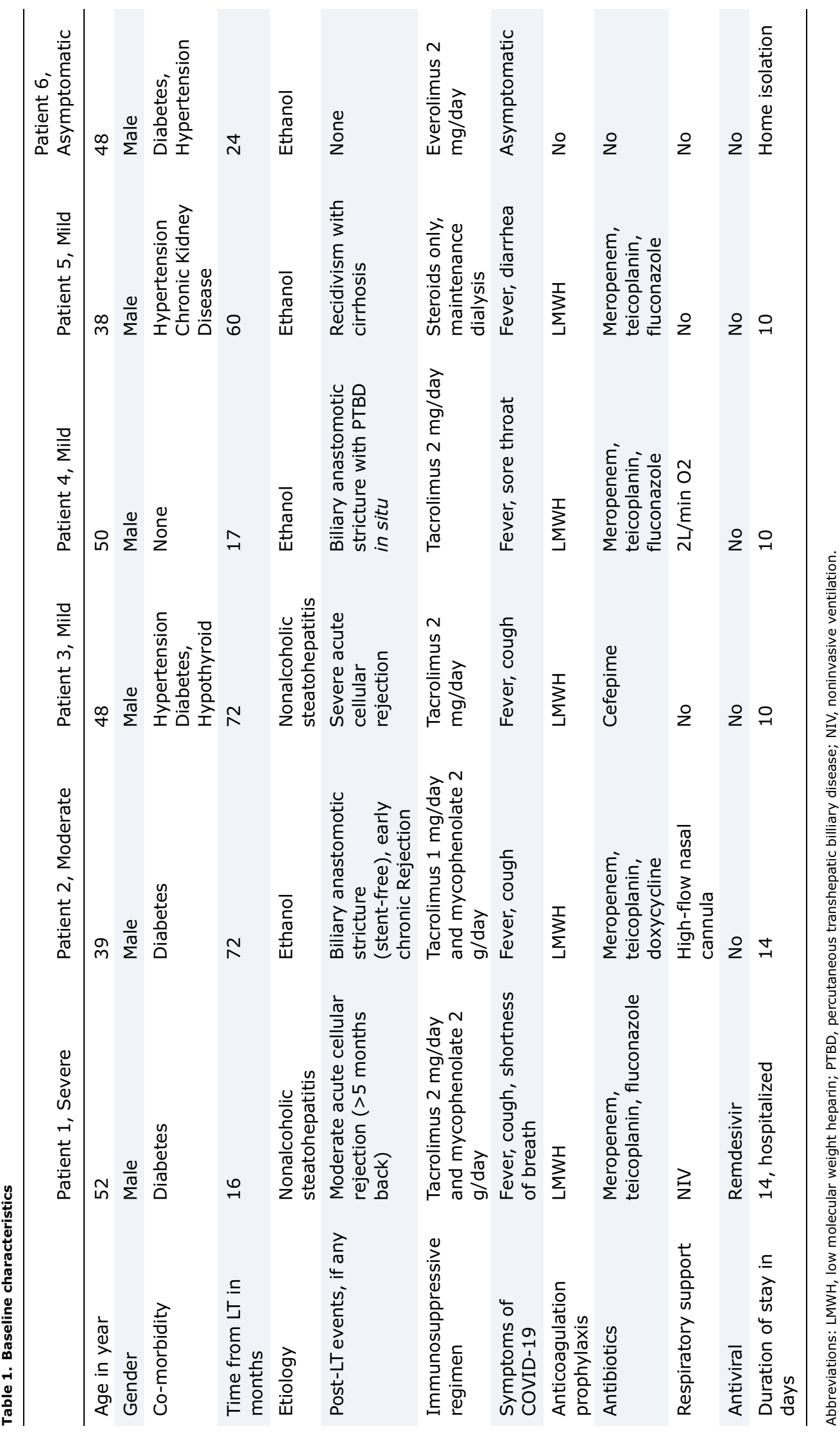




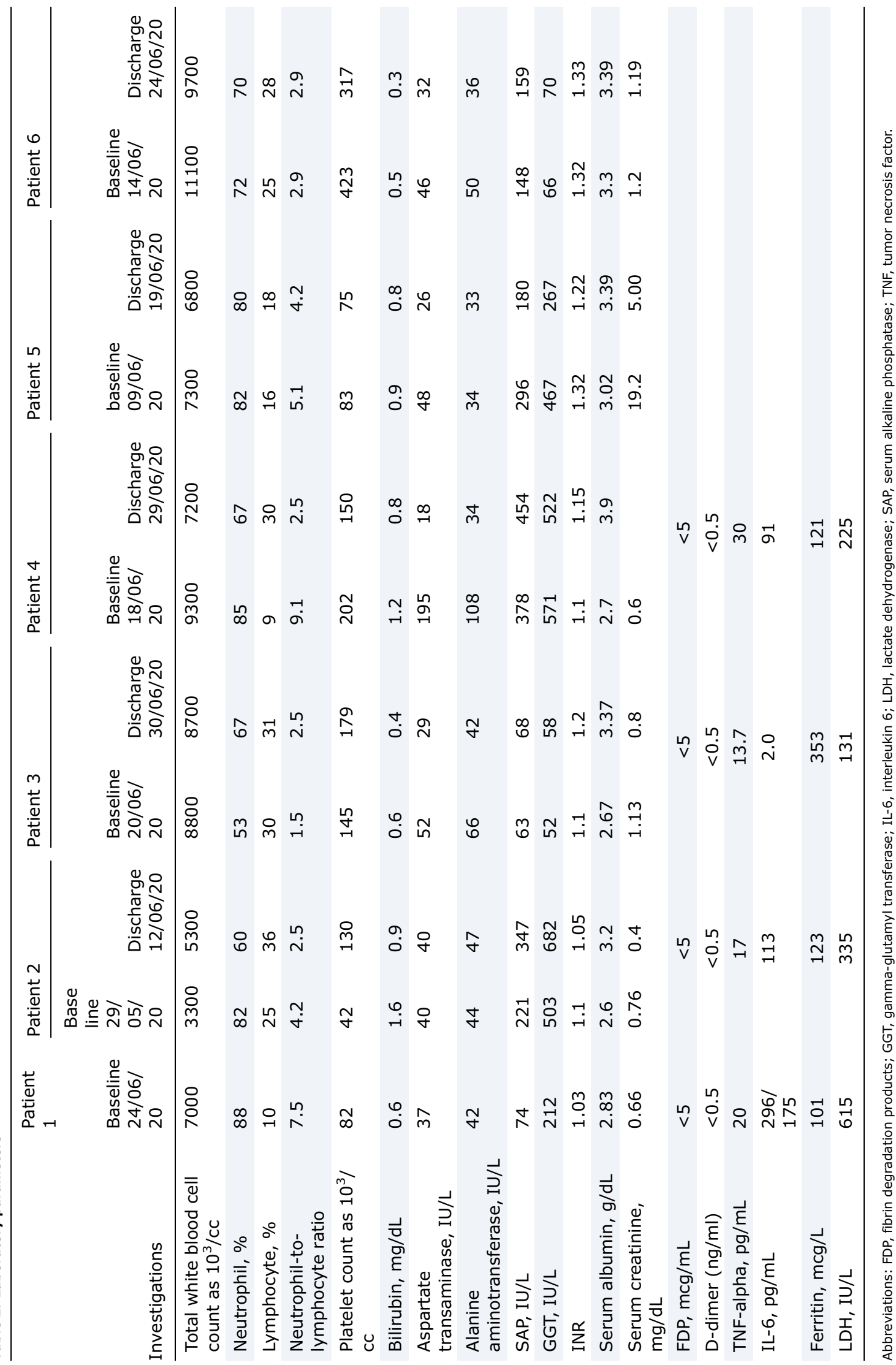



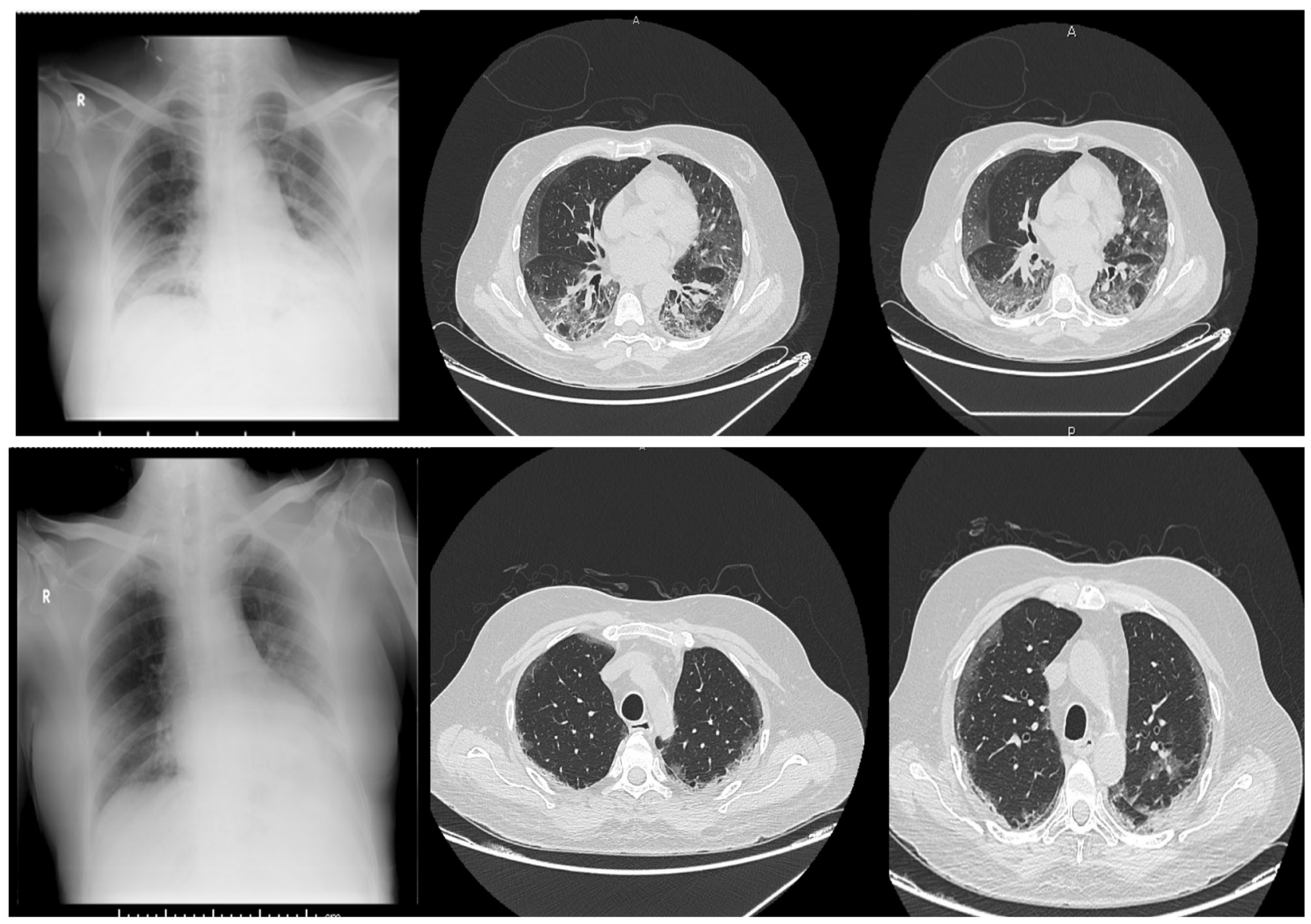

Fig. 1. $X$ ray of chest and CT Thorax at admission (upper panel) and on day 10 (lower panel) which showed improvement, post tocilizumab and convalescent plasma of COVID-19.

\begin{tabular}{|c|c|}
\hline \multicolumn{1}{c|}{ Tacrolimus } & Stopped \\
\hline & Tac \\
\hline Mycophenolate mofetil & Stopped \\
\hline
\end{tabular}

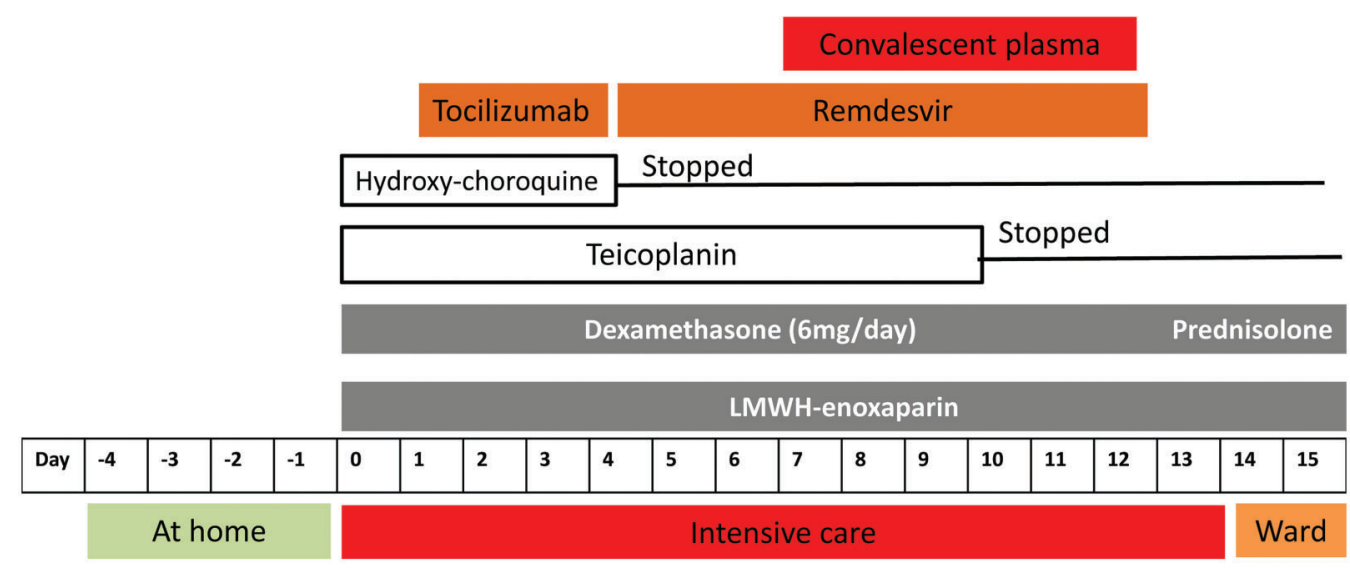

Fig. 2. Treatment timeline of severe CovID-19 case. 
None had deranged liver function, due to immunosuppression minimization for 2 weeks during the COVID-19 infection.

\section{Asymptomatic COVID-19 disease in a post-LT recipient}

This 48 year-old male, 18 months post-LDLT, had a close family contact with a symptomatic COVID-19 case and was asymptomatic positive for SARS-CoV-2 upon testing. He had multiple co-morbidities (i.e. diabetes, hypertension, obesity and had calcineurin inhibitor nephrotoxicity-induced kidney dysfunction, maintained on a calcineurin inhibitor nephrotoxicity-free regimen with everolimus). He was comfortable, afebrile and monitored from home. His everolimus was stopped, and he was given prednisolone (10 mg once daily) without hydroxychloroquine or any COVID-19-specific drug. In view of no symptoms or disease progression, low-dose tacrolimus $(0.5 \mathrm{mg}$ twice daily) and prednisolone ( $5 \mathrm{mg}$ once daily) was started on day 5 , instead of the everolimus. His subsequent SARS-CoV-2 tests, given at two occasions on day 12 and day 14 , were negative. The previous calcineurin inhibitor nephrotoxicity-free regimen (i.e. everolimus) was started on day 15 and there was no derangement of graft function during these period.

\section{Discussion}

The SARS-CoV-2 infection and outcome among solid organ transplant recipients is variable. Whether immunosuppression therapy is a risk is largely unknown, but the severity of disease and outcome has been generally poorer than observed in others. The dosage of immunosuppression at infection and COVID-19 disease severity is poorly correlated. ${ }^{6}$ Most of the recommendations have been for minimization or temporary withdrawal and balance of risk for rejection. However, this modification is individualized but mostly agrees for stopping the antiproliferative drug, reducing or stopping calcineurin inhibitor nephrotoxicity drugs and maintaining on a low dose of steroid. ${ }^{7}$ The same course was followed in the present series and none experienced a rejection.

Severe or moderate COVID-19 often has poor outcome among transplant recipients, with mortality in $12-18 \%$ of cases. ${ }^{8,9}$ The multimodal approach with combination of an antiviral, tocilizumab, has been reported with good outcome. ${ }^{9}$ In our series, the severe case (despite early use of tocilizumab and remdesivir) had a protracted course and addition of convalescent plasma likely helped in recovery for this patient. Convalescent plasma action occurs through binding of the transfused antibodies to the pathogen, resulting in cellular cytotoxicity, phagocytosis, or direct neutralization of the pathogen. ${ }^{10}$ One large study showed that early administration of antibodies led to an optimal clinical effect, as compared to later administration. ${ }^{11}$ However, the data on transplant recipients need to be studied in larger cohorts to determine a routine recommendation.

In the moderate cases of our series, the limited availability of remdesivir or plasma represented a real-world scenario. Consideration of teicoplanin and doxycycline as repurposed drugs helped in the infection's resolution. Teicoplanin is a glycopeptide antibiotic, found to be active in vitro against SARS-CoV in the early stage of the viral life cycle, working by inhibiting the low$\mathrm{pH}$ cleavage of the viral spike protein by cathepsin $L$ and in the late phase by action on the endosomes, thereby preventing the release of genomic viral RNA and causing disruption of the virus replication. ${ }^{12}$ Doxycycline, often used for atypical pneumonia, has been studied recently for SARS-CoV-2 chemoprophylaxis ${ }^{13}$ but regarding its use for therapy the data is scanty. The antiviral effects are secondary to transcriptional up-regulation of the intracellular zinc finger antiviral protein ZAP and repression of RNA translation. ${ }^{13}$ In patients with moderate disease who are transplant recipients with multiple co-morbidities, out more than 2 years after the transplantation, leucopoenia and thrombocytopenia remain challenging scenarios to be managed without tocilizumab or antivirals, like remdesivir. ${ }^{14-16}$

Another important consideration is the natural course of asymptomatic SARS-CoV-2 in a transplant recipient with multiple co morbidities (i.e. diabetes, hypertension and overweight). It was recently shown that 11 of 96 asymptomatic patients developed symptoms, and it was suggested that this could occur more with increasing age. ${ }^{17}$ Kumar et al. ${ }^{18}$ showed the most frequent abnormality in liver functions was hypoalbuminemia, followed by derangements in gamma-glutamyl transferase and aminotransferases, and these abnormalities were more frequent in severe disease. ${ }^{18}$ The results were the same in our study. The consensus is lacking for this group of patients; few have suggested close monitoring, while others in favor of early antiviral therapy to prevent prolong shedding. ${ }^{19}$ Data are limited for treatment in an immunocompromised person or transplant recipients. ${ }^{20}$ The asymptomatic case was managed well by immunosuppression modulation only, with no disease progression or development of symptoms; however, large series are needed to support or refute our findings.

To summarize, we reported six cases of COVID-19 disease in $L T$ recipients with co-morbidities, who were successfully managed. These cases included one severe and one moderate case. Stoppage of antiproliferative or antimetabolites, temporary tacrolimus withdrawal and low-dose maintenance steroid was followed. Severe cases, those with COVID pneumonia, thrombocytopenia or high neutrophil-to-lymphocyte ratio with lymphopenia should be considered for remdesivir and tocilizumab. Convalescent plasma therapy is usually preserved for severe cases in the absence of evidence, mostly for compassionate use or under clinical trial. Therapeutically repurposed drugs with minimal adverse effects, like teicoplanin and doxycycline, can be considered in resource-poor settings.

\section{Funding}

None to declare.

\section{Conflict of interest}

The authors have no conflict of interests related to this publication.

\section{Author contributions}

Conceptualized the study ( $A C$ and SKS); drafted the manuscript (AC), collected the data (GSR, SV), revised the manuscript and generated the figure (VP, SMS, VSTP, RPM, AT, LGM, $\mathrm{DB})$, provided final correction of the manuscript and all logistic support (SKS).

\section{References}

[1] World Health Organization. Coronavirus disease (COVID-19): Weekly epidemiological update. Available from: https://www.who.int/docs/default-source/coronaviruse/situation-reports/20200831-weekly-epi-update-3.pdf? sfvrsn $=\mathrm{d} 7032 \mathrm{a} 2 \mathrm{a}-4$. 
[2] Sarin SK, Choudhury A, Lau GK, Zheng MH, Ji D, Abd-Elsalam S, et al. Preexisting liver disease is associated with poor outcome in patients with SARS CoV2 infection; The APCOLIS Study (APASL COVID-19 Liver Injury Spectrum Study). Hepatol Int 2020:1-11. doi: 10.1007/s12072-020-10072-8.

[3] Belli LS, Duvoux C, Karam V, Adam R, Cuervas-Mons V, Pasulo L, et al. COVID-19 in liver transplant recipients: preliminary data from the ELITA/ELTR registry. Lancet Gastroenterol Hepatol 2020;5:724-725. doi: 10. 1016/S2468-1253(20)30183-7.

[4] Hibi T, Wei Chieh AK, Chi-Yan Chan A, Bhangui P. Current status of liver transplantation in Asia. Int J Surg 2020. doi: 10.1016/j.ijsu.2020.05.071.

[5] COVID-19 India. Available from: https://www.mohfw.gov.in/index1.php.

[6] Pereira MR, Mohan S, Cohen DJ, Husain SA, Dube GK, Ratner LE, et al. COVID-19 in solid organ transplant recipients: Initial report from the US epicenter. Am J Transplant 2020;20:1800-1808. doi: 10.1111/ajt.15941.

[7] Perazzo H, Piedade J, Castro R, Pinto L, Veloso VG, Grinsztejn B, et al. COVID19: An overview of worldwide recommendations for management of patients with liver diseases or liver transplantation. Clin Gastroenterol Hepatol 2020 18:2381-2384.e10. doi: 10.1016/j.cgh.2020.04.074.

[8] Becchetti C, Zambelli MF, Pasulo L, Donato MF, Invernizzi F, Detry O, et al. COVID-19 in an international European liver transplant recipient cohort. Gut 2020: gutjnl-2020-321923. doi: 10.1136/gutjnl-2020-321923.

[9] Lee BT, Perumalswami PV, Im GY, Florman S, Schiano TD. COVID-19 in liver transplant recipients: An initial experience from the US epicenter. Gastroenterology 2020. doi: 10.1053/j.gastro.2020.05.050.

[10] van Erp EA, Luytjes W, Ferwerda G, van Kasteren PB. Fc-mediated antibody effector functions during respiratory syncytial virus infection and disease. Front Immunol 2019;10:548. doi: 10.3389/fimmu.2019.00548.

[11] Jiang J, Miao $Y$, Zhao $Y$, Lu X, Zhou $P$, Zhou $X$, et al. Convalescent plasma therapy: Helpful treatment of COVID-19 in a kidney transplant recipient presenting with serve clinical manifestation and complex complications. Clin Transplant 2020:e14025. doi: 10.1111/ctr.14025.
[12] Zhou N, Pan T, Zhang J, Li O, Zhang X, Bai C, et al. Glycopeptide antibiotics potently inhibit cathepsin I in the late endosome/lysosome and block the entry of ebola virus, middle east respiratory syndrome coronavirus (MERSCoV), and severe acute respiratory syndrome coronavirus (SARS-CoV). J Biol Chem 2016;291:9218-9232. doi: 10.1074/jbc.M116.716100.

[13] Malek AE, Granwehr BP, Kontoyiannis DP. Doxycycline as a potential partner of COVID-19 therapies. IDCases 2020;21:e00864. doi: 10.1016/j.idcr.2020. e00864.

[14] Wang M, Cao R, Zhang L, Yang X, Liu J, Xu M, et al. Remdesivir and chloroquine effectively inhibit the recently emerged novel coronavirus (2019-nCoV) in vitro. Cell Res 2020;30:269-271. doi: 10.1038/s41422-020-0282-0.

[15] Grein J, Ohmagari N, Shin D, Diaz G, Asperges E, Castagna A, et al. Compassionate use of remdesivir for patients with severe covid-19. N Engl J Med 2020;382:2327-2336. doi: 10.1056/NEJMoa2007016.

[16] Nishimoto N, Terao K, Mima T, Nakahara H, Takagi N, Kakehi T. Mechanisms and pathologic significances in increase in serum interleukin-6 (IL-6) and soluble IL- 6 receptor after administration of an anti-IL- 6 receptor antibody, tocilizumab, in patients with rheumatoid arthritis and Castleman disease. Blood 2008;112:3959-3964. doi: 10.1182/blood-2008-05-155846.

[17] Gao Z, Xu Y, Sun C, Wang X, Guo Y, Qiu S, et al. A systematic review of asymptomatic infections with COVID-19. J Microbiol Immunol Infect 2020. doi: $10.1016 /$ j.jmii.2020.05.001.

[18] Kumar-M P, Mishra S, Jha DK, Shukla J, Choudhury A, Mohindra R, et al. Coronavirus disease (COVID-19) and the liver: a comprehensive systematic review and meta-analysis. Hepatol Int 2020:1-12. doi: 10.1007/s12072020-10071-9.

[19] Nacif LS, Zanini LY, Waisberg DR, Pinheiro RS, Galvão F, Andraus W, et al. COVID-19 in solid organ transplantation patients: A systematic review. Clinics (Sao Paulo) 2020;75:e1983. doi: 10.6061/clinics/2020/e1983.

[20] Di Maira T, Berenguer M. COVID-19 and liver transplantation. Nat Rev Gastroenterol Hepatol 2020;17:526-528. doi: 10.1038/s41575-020-0347-z. 